01

\title{
Метод повышения точности расчета характеристик сложных режимов динамики пороговых систем
}

\author{
(C) О.Н. Павлова ${ }^{1}$, А.Н. Павлов ${ }^{1,2,9}$ \\ ${ }^{1}$ Саратовский национальный исследовательский государственный \\ университет им. Н.Г. Чернышевского, Саратов, Россия \\ ${ }^{2}$ Саратовский государственный технический университет \\ им. Гагарина Ю.А., Саратов, Россия \\ ฯE-mail: pavlov.alexeyn@gmail.com
}

Поступило в Редакцию 6 марта 2018 г.

Решается задача повышения точности расчета характеристик сложных режимов динамики пороговых систем на примере показателей Ляпунова. Несмотря на наличие теоретических и численных исследований, ранее позволивших обосновать возможность реконструкции динамических систем по сигналам на выходе пороговых систем, проблема диагностики режимов динамики по малому объему выборки и при наличии помех требует отдельного изучения. Показано, как предварительная обработка данных, предусматривающая переход к равномерной выборке, позволяет значительно уменьшить ошибки вычислений.

DOI: 10.21883/PJTF.2018.15.46442.17279

Расчет показателей Ляпунова является одним из стандартных методов диагностики сложных режимов колебаний нелинейных систем, широко применяемым при решении различных научно-технических задач [1-9]. Расчеты могут проводиться с необходимой точностью, если задана математическая модель исследуемой системы. В экспериментальных исследованиях показатели Ляпунова могут быть вычислены по временно́му ряду при наличии достаточного объема выборки [10]. В целом, чем меньше известно о динамике системы, тем больше погрешность проводимых вычислений. Однако даже при анализе динамики пороговых систем, регистрируемый выходной сигнал которых представляет собой последовательность отсчетов времени $T_{i}$, соответствующих достижению порогового уровня, существует возможность охарактеризовать входной процесс не только на основе методов математической статистики, но 
и с использованием подходов нелинейной динамики. В последние годы данная задача широко обсуждалась в контексте различных нейронных моделей [11]: если на вход модели поступает хаотический сигнал, генерируемый автоколебательной системой, можно ли по последовательности $T_{i}$ вычислить характеристики сложной геометрии и динамики ее аттрактора? Несмотря на то что положительный ответ на этот вопрос был получен для различных типов нейронных моделей [12-15], важным является повышение надежности диагностики режимов динамики. Если существуют ограничения объема выборки и точности регистрации моментов $T_{i}$, то снижение ошибки вычисления характеристик сложных колебаний представляет собой актуальную задачу, один из вариантов решения которой обсуждается в настоящей работе.

Рассмотрим в качестве анализируемой последовательности $T_{i}$ моменты времени пересечения порогового уровня $\theta=1$ хаотическим сигналом $S(t)=x(t)$, который генерируется моделью Ресслера

$$
\frac{d x}{d t}=-(y+z), \quad \frac{d y}{d t}=x+a y, \quad \frac{d z}{d t}=b+z(x-c)
$$

при значениях параметров $a=0.15, b=0.2, c=10$, соответствующих режиму динамического хаоса с показателем Ляпунова $\lambda_{1}=0.087$. В этом случае значения $I_{i}=T_{i+1}-T_{i}$ представляют собой времена возврата фазовой траектории в секущую Пуанкаре. Последовательность времен возврата $I_{i}$ может использоваться в качестве сигнала для реконструкции. Стандартный вариант реконструкции осуществляется путем применения метода задержек непосредственно к последовательности $I_{i}$, т. е. путем создания массива векторов $\mathbf{z}_{i}=\left(I_{i}, I_{i+1}, \ldots, I_{i+m-1}\right)$ в пространстве с размерностью вложения $m$. Чтобы вычислить скорость экспоненциального разбегания траекторий в реконструированном фазовом пространстве, в качестве интервала времени между последовательными векторами $\mathbf{z}_{i}$ выбирается среднее значение времени возврата. Вариант реконструкции, который используется нами, состоит в переходе от значений $T_{i}$ к величинам $S_{r e c}\left(T_{i}\right)=1 /\left(T_{i+1}-T_{i}\right)$, и эти величины далее интерполируются гладкой функцией (кубическим сплайном) для получения сигнала с равномерной выборкой $\Delta t$, а затем к сигналу $S_{r e c}(\Delta t)$ применяется метод задержек [16]. С точки зрения алгоритма расчета показателей Ляпунова [10] такой подход позволяет увеличить количество реконструированных векторов, что способствует снижению ошибок ориентации возмущений в фазовом пространстве

Письма в ЖТФ, 2018, том 44, вып. 15 
при проведении перенормировок [10]. Кроме того, учитываются существующие различия в локальной неустойчивости фазовых траекторий на разных участках, и оценка показателя Ляпунова осуществляется точнее. Чтобы проиллюстрировать это, были проведены расчеты двумя методами при варьировании объема выборки (от 1000 до 3000 отсчетов) и размерности пространства вложения (от 5 до 8). В целях проверки устойчивости метода к флуктуациям в последовательность времен возврата (рис. $1, a$ ) добавлялся белый шум с интенсивностью $1 \%$ от среднего значения времени возврата. На вставке к рис. 1, $a$ показаны моменты пересечения порогового уровня входным сигналом. Результаты представлены в виде распределений вероятности отклонений от ожидаемого значения $\lambda_{1}=0.087$ (рис. $1, b$ ). Как видно из этого рисунка, метод, предусматривающий переход к равномерной выборке, обеспечивает примерно двукратное снижение ошибки вычисления старшего показателя Ляпунова. Этот метод также демонстрирует более высокую устойчивость к аддитивным шумам в анализируемом сигнале.

Отметим, что рассмотренный пример хаотической динамики системы Ресслера является сравнительно простым. Чтобы проверить справедливость сделанных выводов на более сложном примере, рассмотрим хаотический режим пачечной генерации импульсов, демонстрирующий наличие двух различных временны́х масштабов. С этой целью была выбрана модель бета-клетки[17], описываемая следующей системой уравнений:

$$
\begin{gathered}
\frac{d V}{d t}=\left(-I_{\mathrm{Ca}}-I_{\mathrm{K}}-g_{S} S\left(V-V_{\mathrm{K}}\right)\right) / \tau, \\
\frac{d n}{d t}=\mu\left(n_{\infty}-n\right) / \tau, \quad \frac{d S}{d t}=\left(S_{\infty}-S\right) / \tau_{S}, \\
I_{\mathrm{Ca}}(V)=g_{\mathrm{Ca}} m_{\infty}\left(V-V_{\mathrm{Ca}}\right), \quad I_{\mathrm{K}}(V, n)=g_{\mathrm{K}} n\left(V-V_{\mathrm{K}}\right), \\
x_{\infty}=\frac{1}{1+\exp \left(\left(V_{x}-V\right) / \theta_{x}\right)}, \quad x=m, n, S .
\end{gathered}
$$

В проводимых исследованиях рассмотрены следующие значения управляющих параметров: $g_{\mathrm{Ca}}=3.6, g_{\mathrm{K}}=10.0, g_{S}=4.0, \tau=20 \mathrm{~ms}$, $\tau_{S}=35 \mathrm{~s}, \quad V_{\mathrm{Ca}}=25 \mathrm{mV}, \quad V_{\mathrm{K}}=-75 \mathrm{mV}, \quad V_{m}=-20 \mathrm{mV}, \quad V_{n}=-16 \mathrm{mV}$, $V_{S}=-40 \mathrm{mV}, \theta_{m}=12 \mathrm{mV}, \theta_{n}=5.6 \mathrm{mV}, \theta_{S}=10.0 \mathrm{mV}, \mu=0.85$. Peaлизуемый при этом хаотический режим характеризуется показателем Ляпунова $\lambda_{1}=0.011$. В качестве анализируемого сигнала была выбрана

$5^{*}$ Письма в ЖТФ, 2018, том 44, вып. 15 

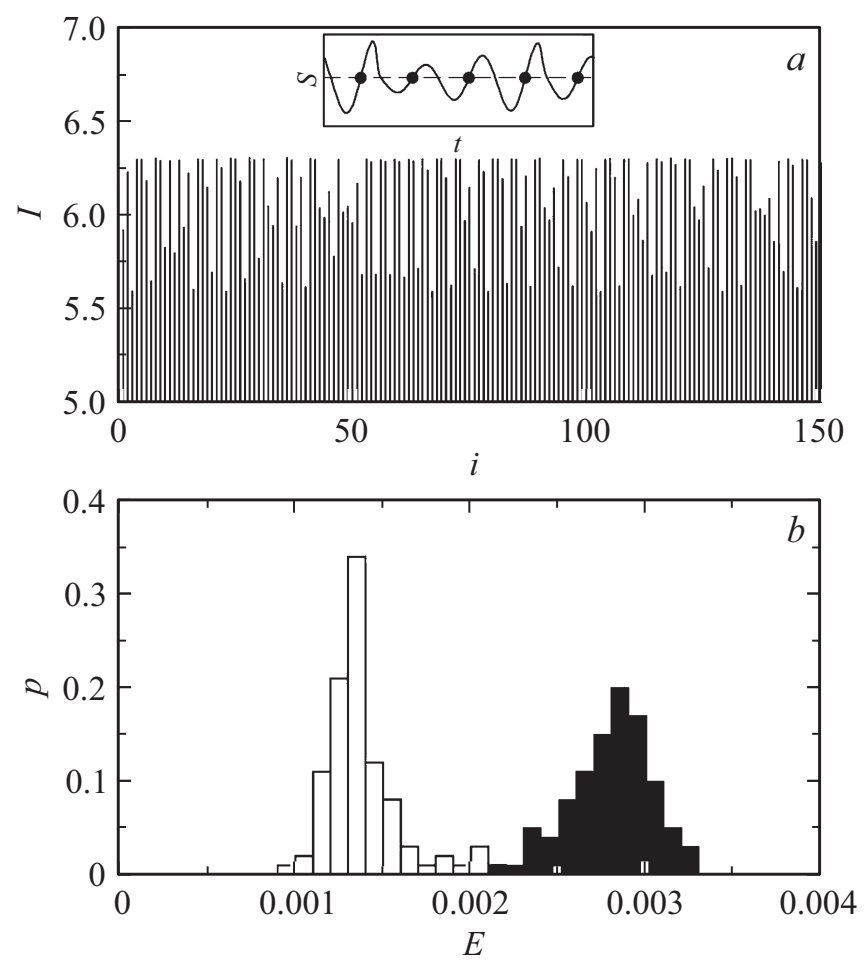

Рис. 1. Последовательность времен возврата хаотического режима динамики модели Ресслера (1) (a) и плотность вероятности отклонений $(E)$ вычисленных значений старшего показателя Ляпунова от ожидаемого значения $\lambda_{1}=0.087$, определенная двумя методами: по исходной последовательности (темные столбики) и после перехода к равномерной выборке (светлые столбики) (b). На вставке показан входной сигнал, кружками отмечены моменты пересечения порогового уровня.

последовательность временны́х интервалов между локальными максимумами сигнала $V(t)$, описывающего напряжение на мембране клетки. По аналогии с предыдущим примером рассматривались последовательности от 1000 до 3000 отсчетов, в которые добавлялся аддитивный белый шум с интенсивностью $1 \%$ от среднего значения временно́го интервала. Результаты приведены на рис. 2, где показан также сигнал

Письма в ЖТФ, 2018, том 44, вып. 15 

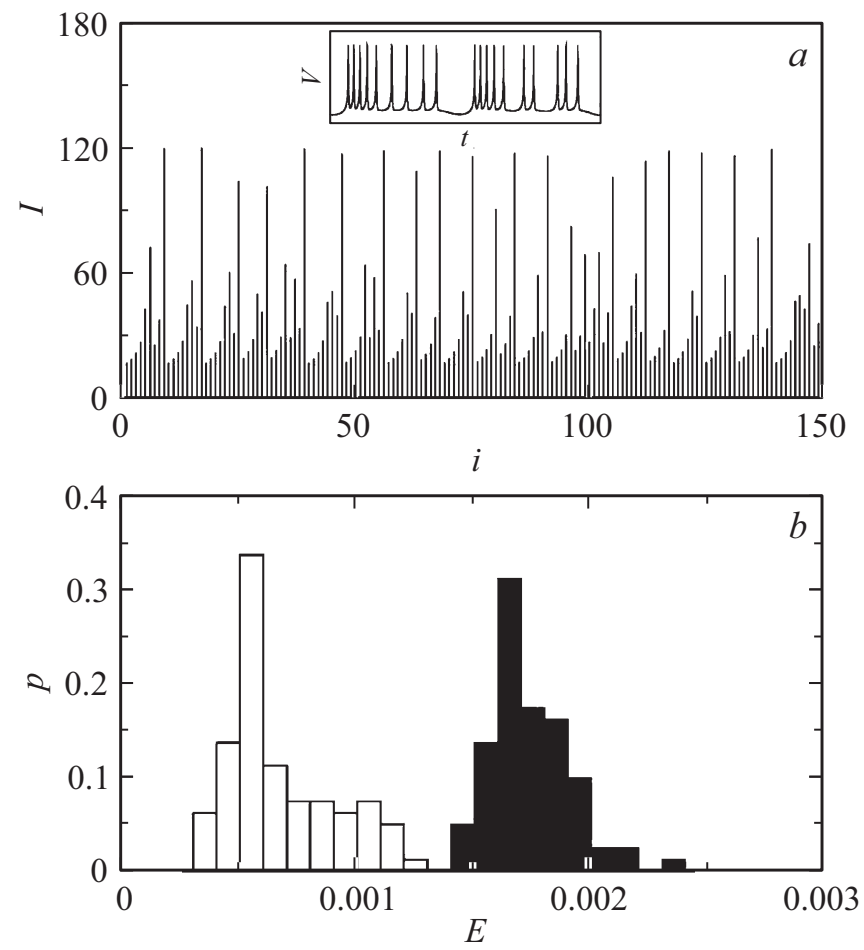

Рис. 2. Последовательность временнб́х интервалов между локальными максимумами сигнала $V(t)$ модели бета-клетки (2) (a) и плотность вероятности отклонений $(E)$ вычисленных значений старшего показателя Ляпунова от ожидаемого значения $\lambda_{1}=0.011$. Обозначения те же, что на рис. 1 . На вставке показана временна́я зависимость переменной $V$.

$V(t)$ (см. вставку на рис. 2,a). Несмотря на усложнение задачи, выводы, сделанные для системы Ресслера, подтверждаются.

Таким образом, проведение предварительного преобразования входного сигнала, предусматривающего переход к равномерной выборке, способствует повышению точности расчета показателей Ляпунова при анализе хаотических режимов колебаний по сигналам на выходе пороговых систем, которое является особенно важным при использовании небольшого объема выборки.

Письма в ЖТФ, 2018, том 44, вып. 15 
Исследование выполнено при поддержке гранта Российского научного фонда (проект № 14-12-00224).

\section{Список литературы}

[1] Потапов А.А., Герман В.А. // Письма в ЖТФ. 2002. Т. 28. В. 14. С. 19-25.

[2] Логунов М.Ю., Бутковский О.Я. // ЖТФ. 2008. Т. 78. В. 8. С. 1-8.

[3] Короновский А.А., Москаленко О.И., Фролов Н.С., Храмов А.Е. // Письма в ЖТФ. 2010. Т. 36. В. 14. С. 19-25.

[4] Москаленко О.И., Павлов А.С. // Письма в ЖТФ. 2014. Т. 40. В. 12. С. 66-72.

[5] Павлов А.Н., Павлова О.Н., Мохаммад Я.Х. // Письма в ЖТФ. 2015. Т. 41. B. 6. C. $98-104$.

[6] Мохаммад Я.Х., Павлова О.Н., Павлов А.Н. // Письма в ЖТФ. 2017. Т. 43. B. 2. C. $45-51$.

[7] Максименко В.А., Постнов Д.Э., Короновский А.А., Макаров В.В., Храмов А.Е. // Письма в ЖТФ. 2017. Т. 43. В. 12. С. 96-103.

[8] Мохаммад Я.Х., Павлова О.Н., Павлов А.Н. // ЖТФ. 2017. Т. 87. В. 11. C. $1753-1755$.

[9] Колоскова А.Д., Москаленко О.И., Короновский А.А. // Письма в ЖТФ. 2018. T. 44. B. 9. С. $19-25$.

[10] Wolf A., Swift J.B., Swinney H.L., Vastano J.A. // Physica D. 1985. V. 16. N 3. P. 285-317.

[11] Daley D.J., Vere-Jones D. An introduction to the theory of point processes. V. 1: Elementary theory and methods. Berlin: Springer, 2003. $471 \mathrm{p}$.

[12] Sauer T. // Phys. Rev. Lett. 1994. V. 72. N 24. P. 3811-3814.

[13] Racicot D.M., Longtin A. // Physica D. 1997. V. 104. N 2. P. 184-204.

[14] Павлов А.Н., Янсон Н.Б., Анищенко В.С. // Радиотехника и электроника. 1999. T. 44. № 9. С. 1075-1092.

[15] Pavlov A.N., Pavlova O.N., Mohammad Y.K., Kurths J. // Chaos. 2015. V. 25. P. 013118

[16] Pavlov A.N., Pavlova O.N., Mohammad Y.K., Kurths J. // Phys. Rev. E. 2015. V. 91. N 2. P. 022921.

[17] Sherman A. // Bull. Mathem. Biology. 1994. V. 56. N 5. P. 811-835. 